\title{
The value of magnetic resonance enterography in diagnostic difficulties associated with Crohn's disease
}

\author{
Katarzyna B. Biernacka ${ }^{1 A, B, E, F}$, Dobromiła Barańska ${ }^{1 B, D}$, Katarzyna Matera ${ }^{1 A, C, D, E}$, Michał Podgórski ${ }^{1 D, E}$, \\ Elżbieta Czkwianianc ${ }^{2 D}$, Katarzyna Szabelska-Zakrzewska ${ }^{2 B, D}$, Inga Dziembowska ${ }^{3 A, C, D}$, Piotr Grzelak ${ }^{1 A, D}$ \\ 'Department of Diagnostic Imaging, Polish Mother's Memorial Hospital - Research Institute, Lodz, Poland \\ 2Department of Gastroenterology, Allergology and Paediatrics, Polish Mother's Memorial Hospital - Research Institute, Lodz, Poland \\ ${ }^{3}$ Department of Pathophysiology, Faculty of Pharmacy, Ludwik Rydygier Collegium Medicum in Bydgoszcz, Nicolaus Copernicus University in Toruń, Poland
}

\section{Abstract}

Purpose: The diagnosis of Crohn's disease (CD), one of the inflammatory bowel diseases (IBD), along with ulcerative colitis (UC), is often challenging due to the limitations of small intestine visualisation. Magnetic resonance enterography (MRE) enables imaging of intraluminal and extraintestinal complications without ionizing radiation. The objective of this study is to select CD-related MRE features and determine the feasibility of this technique to indicate a group of patients that should be subjected to more invasive diagnostic procedures.

Material and methods: A total of 131 patients (mean age 25) underwent MRE, 60 of whom had been previously diagnosed with CD and 17 with UC. Additionally, 26 patients with suspected IBD and 28 with other or unknown pathologies were included in the study. Radiological reports of MRE examinations, effectuated using a 1.5-T field strength, were retrospectively analysed regarding radiological features of IBD, such as the following: bowel wall thickening, enhancement, comb sign, stricture, enlarged mesenteric nodes, inflammatory infiltration, and abnormal diffusion restriction in diffusion-weighted imaging. The statistical model was based on machine learning of the Kohonen map, together with univariate and multivariate analysis.

Results: The selected neuron (Neuron 3) incorporated 23 cases of CD, 9 of suspected IBD, 2 patients with UC, and 4 with other pathologies. The statistical analysis identified bowel wall thickening, intestinal stricture, and lymphadenopathy as the 3 MRE findings most associated with Neuron 3 ( $\mathrm{AUC}=0.919, p=0.031$ ).

Conclusions: Bowel wall thickening, stricture, and enlarged mesenteric lymph nodes in MRE are independent predictive factors for CD diagnosis; thus, patients presenting these features should undergo further examinations. MRE constitutes a powerful imaging modality in cases of suspected IBD.

Key words: inflammatory bowel disease, Crohn's disease, ulcerative colitis, magnetic resonance enterography.

\section{Introduction}

Crohn's disease (CD) is one of the 2 main types of inflammatory bowel disease (IBD), along with ulcerative colitis (UC). The IBD group comprises chronic intestine disorders with not entirely confirmed aetiopathology, involving an autoimmune system impairment [1], a genetic predisposition [2], and an environmental component.
The diagnosis of either $\mathrm{CD}$ or $\mathrm{CU}$ is established based on the clinical manifestation, laboratory tests, imaging techniques, endoscopy, and histopathology results [3]. Thus, patients presenting IBD-related symptoms are subjected to a number of invasive, not well-tolerated diagnostic procedures, such as colonoscopy with histopathologic sample collection. Moreover, a differential diagnosis between the IBD types can be challenging when the histopathological

Correspondence address:

Katarzyna B. Biernacka, Department of Diagnostic Imaging, Polish Mother's Memorial Hospital - Research Institute, 281/289 Rzgowska St, 93-338 Lodz, Poland, phone: +48 42271 15 71, e-mail: kabierka@gmail.com 
results are inconclusive [4,5]. In addition to this, IBD diagnosis, treatment, and follow-up, considering the chronic remitting-relapsing character and the prevalence in the young population, are related to an increased risk of ionizing radiation exposure [6,7]. Depending on the clinical situation, patients with IBD or suspected IBD undergo a variety of ionizing radiological procedures, including plain radiography and computed tomography (CT), as well as nonionizing procedures, such as ultrasonography (US) and magnetic resonance imaging (MRI). Magnetic resonance enterography (MRE) is especially useful in patients with CD because it shows small bowel involvement and transmural inflammation, the 2 main characteristics reserved for this IBD type, which are difficult to evaluate in other radiological and endoscopic exams. The technique of MRE consists of a special imaging protocol with intravenous contrast administration and oral contrast for bowel distension, as opposed to magnetic resonance enteroclysis, which involves a contrast agent administered via nasojejunal tube [8].

The aim of this study is to detect - based on data collected from patients with $\mathrm{CD}, \mathrm{UC}$, as well as IBD suspicion and other diseases - the most characteristic MRE findings for $\mathrm{CD}$, and evaluate the diagnostic potency of magnetic resonance enterography in patients with clinical image of IBD and no histopathological confirmation. The results would help to distinguish, using MRE, among patients with suspected IBD, a group with a higher risk of final CD diagnosis. MRE could be a diagnostic tool to indicate which patient should undergo further studies and be subjected to more invasive diagnostic procedures - colonoscopy or double balloon enteroscopy.

\section{Material and methods}

\section{Patients' characteristics}

A total of 131 MREs were performed in 70 female and 61 male patients ( 67 adults and 64 children), with mean age 25 years, between 2012 and 2018. Among patients submitted to MRE: 60 were previously diagnosed with CD and 17 with ulcerative colitis. The diagnosis was established based on histopathologic samples, obtained during a colonoscopy. Twenty-six patients included in the study were classified as suspected IBD because they were presenting clinical symptoms as well as, in most of the cases, endoscopic findings consistent with IBD, without a definitive histopathological diagnosis. The next, heterogeneous group of 18 cases, listed as other diseases, consisted of the following: other gastrointestinal disorders (familial adenomatous polyposis, diverticulosis, Yersinia enterocolitica infection, and small intestinal bacterial overgrowth syndrome) as well as non-gastroenterological diseases (endometriosis, female infertility unspecified, anorexia nervosa). Finally, the last group of 10 patients, defined as unknown pathologies, presented non-specific symptoms (diarrhoea, abdominal pain, cramping, fatigue, presence of blood in stools, weight loss) and had not been subjected to colonoscopy before MRE.

\section{Magnetic resonance enterography details}

Only exams with a complete, institutionally approved MRE protocol, defined in Tables 1 and 2, were included in the study. A cleansing preparation of the small bowel consisted of a low-residue diet starting 3 days prior to the examination. To avoid artefacts, due to bowel peristalsis, buscolysine $(20 \mathrm{mg} / \mathrm{ml})$ in $1-\mathrm{ml}$ bolus, was administered twice during MRE: the first infusion immediately before the survey scan and the second one directly before diffusion-weighted imaging (DWI), the most sensitive to motion artefacts. Sorbitol and Ispaghula husk solutions were used interchangeably as oral contrast agents. Patients were fasted 6 hours prior to drinking 1.51 of oral contrast solution over a period of 45 minutes up to 1 hour before the examination. In 9 patients, a Gastrografin or Gadoliniumbased MRI contrast agent solution was administered rectally for a better visualisation of distal intestine loops. Two patients, with a history of previous bowel surgeries, had an additional dose of Gastrografin solution injected into the distal loop of ileostomy. MRE was performed in the

Table 1. Magnetic resonance enterography protocol parameters of 1.5-Tesla magnetic field I-VI

\begin{tabular}{|l|c|c|c|c|c|c|}
\hline Parameter & BTFE_SPAIR & T2W_TSE & e-THRIVE & mDIXON & T2_TSE & T2_SPIR \\
\hline Imaging plans & cor & cor & cor & cor & ax & ax \\
\hline TR/TE (ms) & $2 / 1$ & $562 / 80$ & $6 / 2$ & $7 / 0$ & $1.2 / 80$ & $341 / 80$ \\
\hline Flip angle & $80^{\circ}$ & $90^{\circ}$ & $10^{\circ}$ & $15^{\circ}$ & $90^{\circ}$ & $90^{\circ}$ \\
\hline FOV (mm) & 400 & 400 & 400 & 450 & 350 & 350 \\
\hline Selection thickness (mm) & 8.0 & 5.0 & 4.0 & 5.0 & 5.0 & 5.0 \\
\hline Pixel Bandwidth (Hz) & 1578 & 603 & 382 & 619 & 590 & 653 \\
\hline Weighting & $\mathrm{T} 1$ & $\mathrm{~T}$ & $\mathrm{~T} 1$ & $\mathrm{~T}$ & $\mathrm{~T}$ & $\mathrm{~T}$ \\
\hline Acquisition matrix & 288,250 & 240,309 & 200,200 & 232,228 & 252,218 & 252,218 \\
\hline NSA & 1 & 1 & 1 & 1 & 1 & 1 \\
\hline
\end{tabular}

FOV - field of view, NSA - number of signal averages, TE - echo time, TR - repetition time. 
Table 2. Magnetic resonance enterography protocol parameters of 1.5-Tesla magnetic field VII-XIII

\begin{tabular}{|l|c|c|c|c|c|c|c|}
\hline Parameter & DIXON & THRIVE & DWI & c+DIXON & c+DIXON & c+THRIVE & c+THRIVE \\
\hline Imaging plans & $\mathrm{ax}$ & $\mathrm{ax}$ & $\mathrm{ax}$ & $\mathrm{cor}$ & $\mathrm{aX}$ & $\mathrm{cor}$ & $\mathrm{ax}$ \\
\hline TR/TE (ms) & $5 / 0$ & $4 / 2$ & $1.5 / 75$ & 7.0 & $5 / 0$ & $6 / 2$ & $4 / 2$ \\
\hline Flip angle & $15^{\circ}$ & $10^{\circ}$ & $90^{\circ}$ & $15^{\circ}$ & $15^{\circ}$ & $10^{\circ}$ & $10^{\circ}$ \\
\hline FOV (mm) & 350 & 384 & 400 & 450 & 350 & 400 & 380 \\
\hline Selection thickness (mm) & 5.0 & 4.0 & 6.0 & 5.0 & 5.0 & 4.0 & 4.0 \\
\hline Pixel Bandwidth (Hz) & 723 & 434 & 2216 & 619 & 723 & 382 & 432 \\
\hline Weighting & $\mathrm{T} 1$ & $\mathrm{~T} 1$ & Diffusion & $\mathrm{T} 1$ & $\mathrm{~T} 1$ & $\mathrm{~T} 1$ & $\mathrm{~T} 1$ \\
\hline Acquisition matrix & 268,206 & 216,216 & 132,132 & 232,228 & 268,206 & 200,200 & 223,224 \\
\hline NSA & 1 & 1 & 2 & 1 & 1 & 1 & 1 \\
\hline Intravenous contrast & No & No & No & Yes & Yes & Yes & Yes \\
\hline
\end{tabular}

FOV - field of view, NSA - number of signal averages, TE - echo time, TR - repetition time, TSE - turbo spin echo.

prone position with feet first on a 1.5-T MR system (Ingenia Omega HP, Philips Healthcare Nederland) equipped with a dedicated multi-channel body array coil (dStream Torso, 32 channels, Philips Healthcare Nederland). Tables 1 and 2 contain a detailed description of the MRE protocol. A Gadolinium-based agent (Gadovist, Prohansen, or Dotarem) at a dose of $0.1 \mathrm{mmol} / \mathrm{kg}$ was administered intravenously. A contrast sequence was preceded by a DWI scan with double b-values: 100 and $800 \mathrm{~s} / \mathrm{mm}^{2}$. All sequences were covering the abdomen and pelvis, from the lung bases to the pubic symphysis. All MR scans used breath hold, except for the balanced turbo field echo (BTFE), where a triggering breath hold function was used. Thus, all sequences used a parallel imaging (sensitivity encoding - SENSE) factor for scan time reduction.

\section{Statistical analysis}

Radiological reports of 131 MREs, assembled in the following groups: 17 with diagnosed UC, 60 with CD, 26 with IBD suspicion, named as IBD unsure, 18 with other diseases, and 10 with unknown pathologies, were retrospectively inspected considering typical CD enterography features. 126 of all the 131 MREs were evaluated by the same radiologist with 20 years' experience, specialized in MRI assessment of digestive tract diseases. Only 5 exams were interpreted by different diagnostic imaging specialists, but they were supervised by the mentioned-above radiologist as a consultant. All the radiologists used the same notions to describe MRE findings, as shown in Table 3. Additionally, MRE reports were analysed regarding the extent of the disease, as the following parts of intestine affected by inflammatory process, were identified: jejunum, ileum, cecum, ascending colon, transverse colon, descending colon, sigmoid colon, rectum, anal canal, as well as appendix and ileocecal valve. The specialists were not blinded to the previous CD or UC diagnosis. The statistical analysis was performed using Statistica 13.3 (StatSoft, Cracow, Poland).
A Kohonen neural network was applied to organize all the features described in MRE reports (Table 3). Kohonen maps are based on natural neural networks, which enable grouping data from different sources. The network, provided with input data, representing raw, unprocessed information (in this case MRE features), recognizes relationships occurring in clusters and categorizes them in a self-learning process. The benefit of the data sample is enhanced if the compounds are diverse, because the network is able to group and distinguish inputs more efficiently. Thus, the data collected from all the groups of patients: diagnosed with CD and UC, with suspected IBD, as well as the patients with other diseases and unknown pathologies, were included in input data. Due to the ability to self-organize and adapt to formerly unknown information [17], as a result of processing all the input data, the Kohonen map enabled to determine $1 \times 3=3$ classes of data ( 3 neurons), representing 3 groups of patients, categorized based on MRE features. A $\chi^{2}$ test and a Fisher's exact test were used to compare the distribution of predictions between neurons. Based on the conducted tests, one neuron (Neuron 3) was selected as the one that most closely corresponded with the sample input, incorporating the most patients with $\mathrm{CD}$ diagnosis. Univariate analysis was used to examine the relationship between the Neuron 3 and radiological features of CD, defined in Table 3. Furthermore, the association between the neuronal model and abnormal diffusion restriction was analysed, as well as the presence of MRE features in each of the defined and described above digestive tract locations. Parameters proven to be significant on the univariate analysis, with $\mathrm{p}$-values inferior to 0.10 , were afterwards tested on multivariate model. The high threshold p-level was established to reduce the risk of failing to acknowledge a relationship in the data and to reject the variables with a weak association when assessed alone, which could demonstrate significance, when combined with other variables. The actual $p$-values for all the selected variables were inferior to 0.05 . 
Table 3. Inflammatory bowel disease features in magnetic resonance enterography (MRE)

\begin{tabular}{|c|c|}
\hline $\begin{array}{l}\text { Radiological feature } \\
\text { in MRE }\end{array}$ & Description and criteria \\
\hline Mural thickening & Bowel wall greater than $3 \mathrm{~mm}[9]$ \\
\hline Mural enhancement & $\begin{array}{l}\text { Increased signal intensity in a bowel wall after a contrast application (in post-gadolinium T2 images) when compared to } \\
\text { the adjacent bowel loops }[10,11]\end{array}$ \\
\hline Intraperitoneal free fluid & Presence of free fluid in the peritoneal cavity, including a subtle pelvic fluid collection in the pouch of Douglas \\
\hline Fibro-fatty proliferation & $\begin{array}{l}\text { Pseudo-mass adjacent to the bowel loop involved in inflammatory process, with signal characteristics consistent with } \\
\text { fibrous or/and fatty tissue [9] }\end{array}$ \\
\hline Increased vascularity & Prominent pericolic or perienteric vasculature \\
\hline Comb sign & Engorgement: dilated, tortuous, and elongated appearance of vasa recta $[9,12]$ \\
\hline Hyperaemia & Increased blood flow in intestinal wall and adjacent tissues demonstrated as hyperenhancement \\
\hline Stricture & Narrowing of the bowel lumen when compared to adjacent segments \\
\hline Obstruction & Complete luminal obstruction of the bowel \\
\hline Mesenteric adenopathy & Presence of mesenteric lymph nodes exceeding $5 \mathrm{~mm}$ in transverse diameter [13] \\
\hline Abscess & Well-defined fluid collection with an air-fluid level (Figure 1) \\
\hline Fluid collection & Fluid collection with a thin, smooth capsule, without a rim enhancement or an air-fluid level \\
\hline Fistula & Fluid containing enhancing tracts communicating with adjacent structures or a star-sign presentation (Figure 2) \\
\hline $\begin{array}{l}\text { Inflammatory } \\
\text { infiltration }\end{array}$ & $\begin{array}{l}\text { Coexistence of at least } 3 \text { of the listed features: mural enhancement, mural thickening, intraperitoneal free fluid, increased } \\
\text { vascularity, comb sign, hyperaemia, fluid collection, abnormal diffusion restriction }\end{array}$ \\
\hline Colonic haustral loss & Disappearance of colonic haustral folds \\
\hline $\begin{array}{l}\text { Bauhin valve } \\
\text { abnormalities }\end{array}$ & $\begin{array}{l}\text { Thickened lips - valve lips exceeding } 5 \mathrm{~mm}[14] \\
\text { Dilatation and valve's insufficiency - defective valve's opening, resulting in widened valve's lumen } \\
\text { Fibrosis - stricture with the signal characteristics consistent with fibrous tissue and no enhancement } \\
\text { Valve resection } \\
\text { Stricture - narrowed valve lumen } \\
\text { Inflammatory infiltrate - enhanced and thickened valve lips with adjacent mesenteric enhancement and hyperaemia } \\
\text { Atypical shape - loss of normal ileocecal valve morphology }\end{array}$ \\
\hline $\begin{array}{l}\text { Abnormal diffusion } \\
\text { restriction }\end{array}$ & $\begin{array}{l}\text { Hyperintensity on DWI images corresponding with low ADC (apparent diffusion coefficient) values - comparing to adjacent } \\
\text { loops }[15,16]\end{array}$ \\
\hline
\end{tabular}

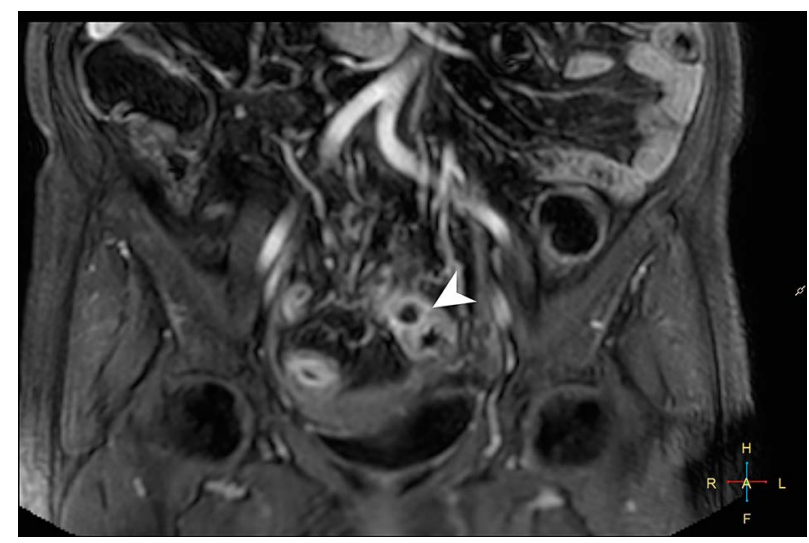

Figure 1. Magnetic resonance enterography mDIXON coronal scan of a Crohn's disease patient, showing an inflammatory infiltrate of sigmoid wall, sigmoid mesocolon, and surrounding adipose tissue with an abscess

For the multivariate model, a forward selection procedure, considering the parameter of area under curve (AUC), was used to obtain significant independent factors of CD diagnosis. In all other analyses, $p<0.05$ was considered as significant.

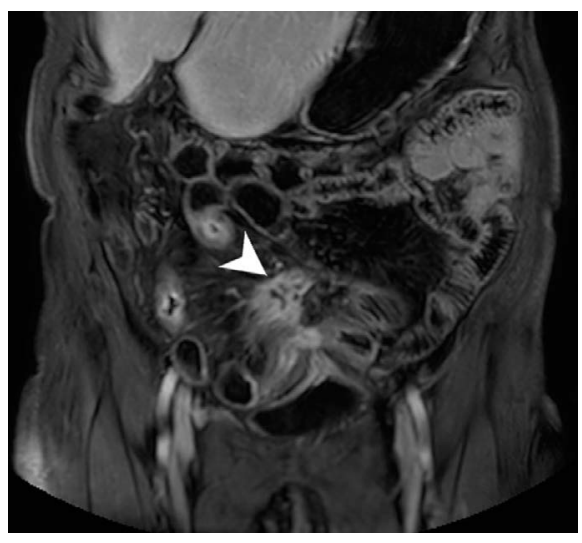

Figure 2. Magnetic resonance enterography mDIXON coronal scan of a Crohn's disease patient presenting a fistula between adjacent intestinal loops manifested as a star-sign

\section{Results}

Among 38 cases incorporated in the Neuron 3, 23 were represented by patients with $\mathrm{CD}, 9$ cases by unsure IBD, 2 by patients with $\mathrm{UC}$, a single person with unknown pa- 
thology, and 3 cases of other diseases. Univariate analysis of MRE features associated with Neuron 3, considering the parameters of the odds ratio (OR) and $p$-values, included the following: bowel wall thickening $(\mathrm{OR}=15.19$, $p<0.001)$, presented in Figure 3, bowel wall enhancement $(\mathrm{OR}=12.00, p<0.001)$, comb sign $(\mathrm{OR}=7.00$, $p=0.020)$, stricture $(\mathrm{OR}=10.12, p<0.001)$, enlarged mesenteric nodes $(\mathrm{OR}=6.09, p<0.001)$, inflammatory infiltration ( $\mathrm{OR}=29.74, p=0.002)$, and Bauhin's valve thickened lips $(\mathrm{OR}=0.18, p=0.010)$. Other factors, subjected to analysis with $95 \%$ confidential index $(\mathrm{CI})$, were: free fluid, fibrofatty proliferation, increased vascularity, hyperaemia, abscess, fluid collection, fistula, and colonic haustral loss. For these characteristics, the type 1 error was greater than 5\%. A multivariate analysis selected using the forward selection procedure (AUC $=0.919, p=0.031$ ) identified bowel wall thickening, intestinal stricture, and lymphadenopathy as the $3 \mathrm{MRE}$ features the most associated with Neuron 3.

The statistical analysis did not indicate any bowel part as associated with Neuron 3. Seemingly abnormal diffusion restriction was not proven to be related with Neuron 3: $\mathrm{OR}=2.57$ and $p=0.110(\mathrm{CI}=95 \%)$.

\section{Ethical considerations}

The study was given a favourable opinion by the independent Bioethical Committee of the Polish Mother's Memorial Hospital Research Institute (the number of the approval 105/2018). Written informed consent was obtained from each of the patients before MRE.

\section{Discussion}

\section{Principal outcomes of the study}

Given the considerable number of patients with $C D$, incorporated in the Neuron 3 (23 cases), we came to the conclusion that the chosen neuron was the most CDspecific. Thus, bowel wall thickening, intestinal stricture, and lymphadenopathy, which most associated with the Neuron 3, were interpreted as the most significant predictive factors for CD diagnosis. Conversely, neither abnormal diffusion restriction, nor localisation of MRE changes in any part of the bowel, were proven to be predictive factors of Crohn's disease.

Bowel wall thickening and intestinal stricture, detected by the statistical analysis as the most associated with CD diagnosis, are indisputably common findings in CD patients $[18,19]$, due to the inflammatory cell infiltration, fibrosis, and the transmural character of the disease. Thus, our study results were along expected lines, but none of these findings is specific for active disease. The statistical analysis did not prove DWI restriction (Figure 4), considered as an active disease finding [16,20], to be a predictive factor for CD diagnosis. Multiple studies evaluated the use

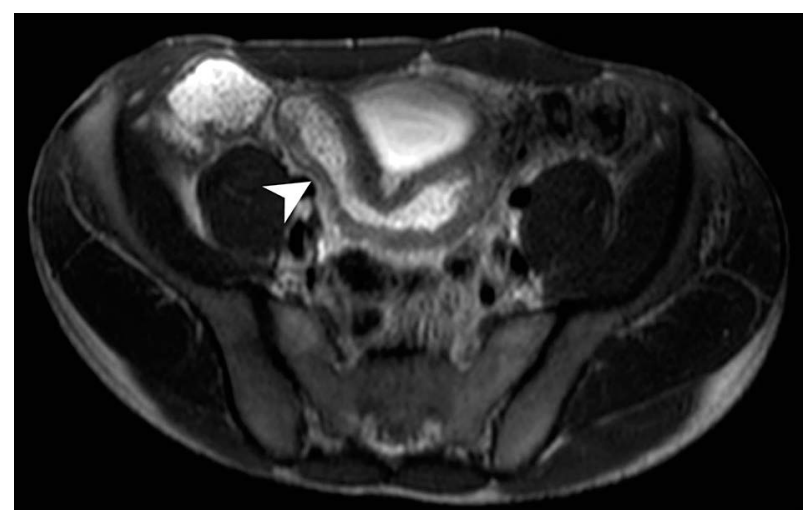

Figure 3. Magnetic resonance enterography axial scan in $\mathrm{T} 2 \mathrm{~W}$ of a patient with suspected inflammatory bowel diseases, demonstrating ileum wall thickening

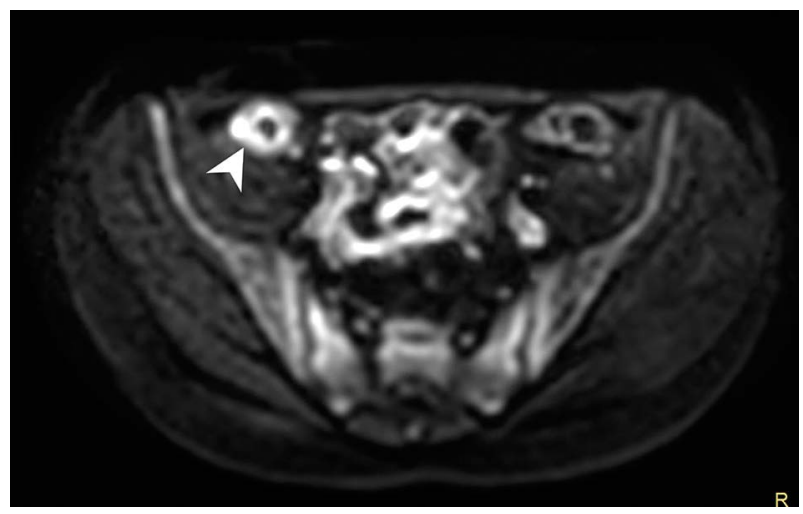

Figure 4. Magnetic resonance enterography axial scan, $b$-value 800 , showing abnormal diffusion restriction in Crohn's disease involved ileum

of MRE in the differentiation between active and inactive CD $[8,13]$. Athanasakos et al. suggested that chronic disease lesions are represented by thickened bowel wall with homogenous and slow enhancement on delayed post-contrast injection T1W images, together with a low intensity on $\mathrm{T} 2 \mathrm{~W}$ fat saturation images. Whereas, as proposed in their study, acute active CD demonstrates with an intense and early mucosal enhancement and a following transmural enhancement on post-gadolinium T1W scans. According to Albert et al. bowel wall enhancement should be considered as an active small bowel CD feature only if coexisting with a thickening of the bowel wall, otherwise it must be interpreted as non-specific [21]. In our study, mural enhancement was defined as an increased signal intensity in a bowel wall, in post-gadolinium T2 images, when compared to the adjacent bowel loops. The pattern and the evolution of the enhancement were not described in the radiological reports. What is more, the radiological findings, separately classified as follows: mural enhancement, increased vascularity, comb sign, and hyperaemia, are morphological presentations of the same CD characteristic. Thus, the analysis of MRE reports demonstrates a need for standardization of MRE description to avoid redundancy and to provide information concerning the pattern and evolution of the enhancement for a better 


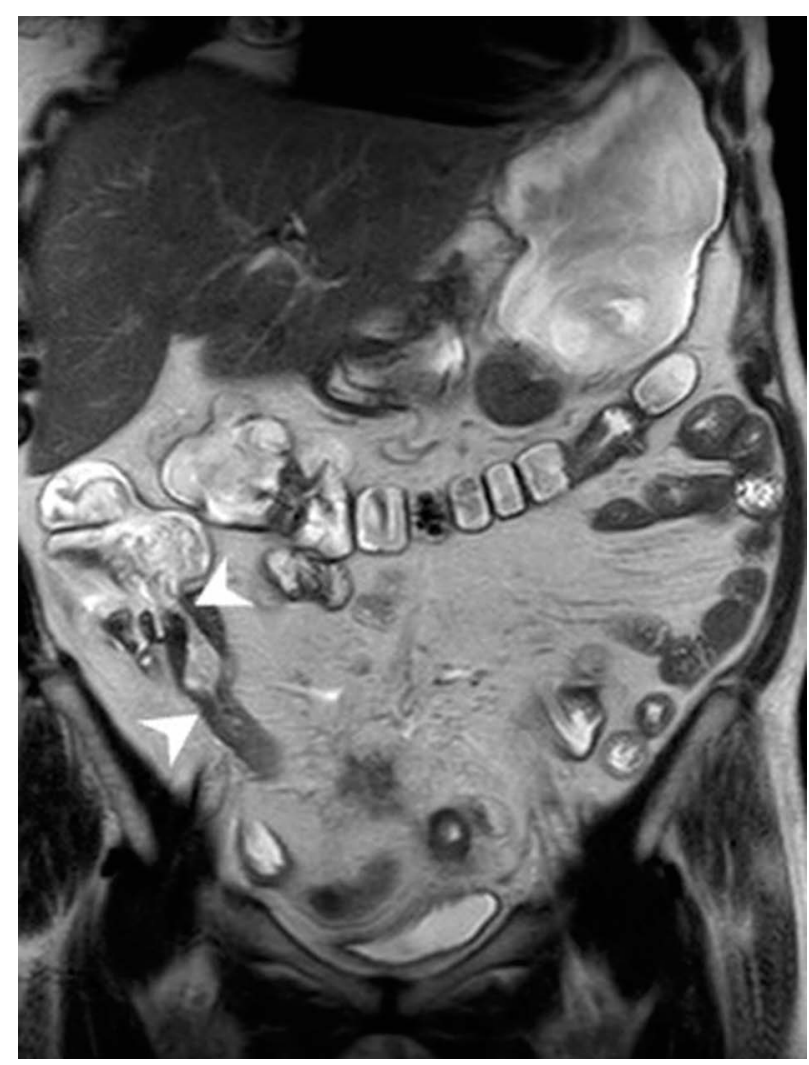

Figure 5. Magnetic resonance enterography scan in T2W TSE coronal BH sequence of a patient with Crohn's disease, showing thickened lips and Bauhin valve stricture as well as thickened wall and stricture of terminal ileum

distinction between active and fibrotic lesions. Another issue crucial for the $\mathrm{CD}$ diagnosis concerns the interpretation of intestinal stricture. In multiple studies [10,22,23] an upstream dilation coexisting with a luminal narrowing is obligatory for recognition of the stricture. The abovementioned recommendations [23] pointed out a possibility of misinterpretation of a bowel spasm as a stricture. However, research by Lunder et al. defined intestinal stricture as a reduction of at least $80 \%$ in the bowel lumen compared with an adjacent loop [24]. Albert et al. described a significant intestinal stricture as a narrowing of the small bowel lumen to less than $12 \mathrm{~mm}$ [21]. In both of the above-cited studies, detection of an upstream dilation was not mandatory for stricture recognition. In our study, a proximal dilation was not included in the stricture definition; thus, the outcome of MRE evaluation was subject to the bias of false positive findings.

Additionally, the results of our study did not distinguish a localisation of inflammatory changes in any of the above-defined bowel parts, as associated with CD diagnosis. The ileocecal region (Figure 5), with the distal segment of the small bowel, is known to be the most frequent Crohn's disease extent $[22,25,26]$. The lack of confirmation of terminal ileum changes as the predictive factor for CD may result from a large group of patients with other or non-defined pathologies (28 cases), a potentially great number of patients with no IBD, which would bias the results.
An interdisciplinary study conducted by Bruining et al. [23] led to the formulation of guidelines for CT and MRE in patients with small bowel CD. A proper establishment of cut-off values constitutes a difficulty related to bowel wall thickening assessment. The consensus [23] defined the abnormal thickness of bowel wall as $3 \mathrm{~mm}$ and more, which corresponds with our definition (bowel wall greater than $3 \mathrm{~mm}$ considered as thickened), as well as the one formulated in the study by Athanasakos et al. [13]. The estimation of the cut-off point is also challenging regarding dimensions of mesenteric nodes. In the reported recommendations, lymphadenopathy is defined as the presence of lymph nodes greater than $1.5 \mathrm{~cm}$ in the short axis. In our analysis, mesenteric lymph nodes were considered enlarged when exceeding $0.5 \mathrm{~cm}$ in the short axis, which is identical to the lymphadenopathy definition used by other researchers [13]. The divergence of measurement method may result in large differences in the interpretation of CD findings. Thus, a significant advantage of our study is that a great majority of MREs were evaluated, and all of them were verified by the same experienced radiologist. Moreover, the specialists were using the same nomenclature to describe the IBD-related MRE findings. Therefore, the conclusion can be drawn that the magnetic resonance characteristics of IBD found and named by the radiology specialist were unequivocal.

\section{Research limitations}

Some limitations of the study should be noted. Firstly, the radiologists, while creating the MRE reports, were not blinded to the histopathology results, confirming CD diagnosis. Hence, for the group of patients with diagnosed Crohn's disease, there is a risk of bias because the specialists could be more inclined to find CD-related MRE features in these patients. However, the radiologists did use the same system of notions and criteria, regardless of the given diagnosis. Secondly, given the small group of patients, data used to create the neuronal model was reduced. A substantial number of patients were diagnosed with other diseases [18], which do not exclude a coexisting, undiagnosed IBD. Furthermore, the group of UC patients was limited. This is due to the fact that ulcerative colitis affects only the large bowel, which can be fully examined in colonoscopy. Thus, among the MRE indications, the cases of UC are significantly less often presented than Crohn's disease. The self-organizing map, in order to develop meaningful clusters, requires correct and sufficient information. Incomplete or non-representative input data may result in an inaccurate outcome. Moreover, given the retrospective character, additional patients' information, including detailed clinical presentation and laboratory test results if performed in other medical establishments, are not available. Consequently, the comparison of MRE results and the Mayo Clinic Score for UC or Crohn's Disease Activity Index (CDAI) [22] was impossible. 
Another constraint concerns the lack of endoscopic and histopathological studies performed within a few weeks before or after MRE for a large number of patients. The time interval between colonoscopy with sample collection and MRE was often too long to consider the 2 modalities as performed in the same clinical situation. A comparative analysis of the 2 diagnostic techniques performed within the maximal interval of 4 weeks would be of a great value, as demonstrated by the researchers from Clermont Auvergne University [27]. According to the results of their study, a deep MRI remission defined using the Barcelona as well as Clermont criteria correlates with mucosal healing in endoscopy with specificity, respectively, of $85.3 \%$ and $88.2 \%$, as well as negative predictive values of, respectively, $85.3 \%$ and $85.7 \%$.

\section{Conclusions}

Magnetic resonance enterography constitutes a valuable diagnostic tool in patients with suspected IBD without a confirmed diagnosis. Bowel wall thickening, intestinal stricture, and lymphadenopathy are observed in patients more inclined toward CD diagnosis, who should undergo further, more invasive diagnostic procedures. Distinguishing between active and chronic disease remains a challenge. There is a need to search for the best MRE finding to differentiate between fibrostenotic and active lesions in Crohn's disease. MRE confirms its utility in differential diagnosis between CD and UC, demonstrating small bowel involvement, transmural character, and extraintestinal complications of Crohn's disease. The MRE evaluation should be improved and standardized to create a consistent and explicit reporting system for enterography findings in order to estimate disease severity and facilitate therapeutic decisions.

\section{Conflict of interest}

The authors report no conflict of interest.

\section{References}

1. Byndloss MX, Litvak Y, Bäumler AJ. Microbiota-nourishing immunity and its relevance for ulcerative colitis. Inflamm Bowel Dis 2019; 25: 811-815.

2. Wang MH, Fiocchi C, Ripke S, et al. A novel approach to detect cumulative genetic effects and genetic interactions in Crohn's disease. Inflamm Bowel Dis 2013; 19: 1799-1808.

3. Maaser C, Sturm A, Vavricka SR, et al. ECCO-ESGAR guideline for diagnostic assessment in IBD. Part 1: Initial diagnosis, monitoring of known IBD, detection of complications. J Crohns Colitis 2019; 13: $144-164$.

4. Magro F, Langner C, Driessen A, et al. European consensus on the histopathology of inflammatory bowel disease. J Crohns Colitis 2013; 7: 827-851.

5. Levine A, Koletzko S, Turner D, et al. ESPGHAN revised porto criteria for the diagnosis of inflammatory bowel disease in children and adolescents. J Pediatr Gastroenterol Nutr 2014; 58: 795-806.

6. Anupindi SA, Grossman AB, Nimkin K, et al. Imaging in the evaluation of the young patient with inflammatory bowel disease: what the gastroenterologist needs to know. J Pediatr Gastroenterol Nutr 2014; 59: 429-439.

7. Zakeri N, Pollok RC. Diagnostic imaging and radiation exposure in inflammatory bowel disease. World J Gastroenterol 2016; 22: 2165 2178.

8. Gee MS, Harisinghani MG. MRI in patients with inflammatory bowel disease. J Magn Reson Imaging 2011; 33: 527-534.

9. Mentzel HJ, Reinsch S, Kurzai M, et al. Magnetic resonance imaging in children and adolescents with chronic inflammatory bowel disease. World J Gastroenterol 2014; 20: 1180-1191.

10. Alexopoulou E, Roma E, Loggitsi D, et al. Magnetic resonance imaging of the small bowel in children with idiopathic inflammatory bowel disease: evaluation of disease activity. Pediatr Radiol 2009; 39: 791-797.
11. Qiu Y, Mao R, Chen BL et al. Systematic review with meta-analysis: magnetic resonance enterography vs. computed tomography enterography for evaluating disease activity in small bowel Crohn's disease. Aliment Pharmacol Ther 2014; 40: 134-146.

12. Lee SS, Kim AY, Yang SK, et al. Crohn disease of the small bowel: comparison of CT enterography, MR enterography, and small-bowel follow-through as diagnostic techniques. Radiology 2009; 251: 751-761.

13. Athanasakos A, Mazioti A, Economopoulos N, et al. Inflammatory bowel disease-the role of cross-sectional imaging techniques in the investigation of the small bowel. Insights Imaging 2015; 6: 73-83.

14. Bogers JJ, Van Marck E. The ileocaecal junction. Histol Histopath 1993; 8: 561-566.

15. Jesuratnam-Nielsen K, Løgager VB, Munkholm P, et al. Diagnostic accuracy of three different MRI protocols in patients with inflammatory bowel disease. Acta Radiol Open 2015; 4: 2058460115588099.

16. Pendsé DA, Makanyanga JC, Plumb AA, et al. Diffusion-weighted imaging for evaluating inflammatory activity in Crohn's disease: comparison with histopathology, conventional MRI activity scores, and faecal calprotectin. Abdom Radiol (NY) 2017; 42: 115-123.

17. Kohonen T. Essentials of the self-organizing map. Neural Netw 2013; 37: 52-65.

18. Makanyanga JC1, Pendsé D, Dikaios N et al. Evaluation of Crohn's disease activity: initial validation of a magnetic resonance enterography global score (MEGS) against faecal calprotectin. Eur Radiol 2014; $24: 277-287$.

19. Bessissow T, Reinglas J, Aruljothy A et al. Endoscopic management of Crohn's strictures. World J Gastroenterol 2018; 24: 1859-1867.

20. Dubron C, Avni F, Boutry N, et al. Prospective evaluation of free-breathing diffusion-weighted imaging for the detection of inflammatory bowel disease with MR enterography in childhood population. Br J Radiol 2016; 89: 20150840. 
21. Albert JG, Martiny F, Krummenerl A et al. Diagnosis of small bowel Crohn's disease: a prospective comparison of capsule endoscopy with magnetic resonance imaging and fluoroscopic enteroclysis. Gut 2005; 54: 1721-1727.

22. Sturm A, Maaser C, Calabrese E, et al. ECCO-ESGAR guideline for diagnostic assessment in IBD. Part 2: IBD scores and general principles and technical aspects. J Crohns Colitis 2019; 13: 273-284.

23. Bruining DH, Zimmermann EM, Loftus EV Jr, et al. Consensus recommendations for evaluation, interpretation, and utilization of computed tomography and magnetic resonance enterography in patients with small bowel Crohn's disease. Radiology 2018; 286: 776-799.

24. Lunder AK, Bakstad LT, Jahnsen J, et al. Assessment of bowel inflammation and strictures by magnetic resonance enterography in long-term Crohn's disease. J Crohns Colitis 2019; 13: 607-614.

25. Goulart RA, Barbalho SM, Gasparini RG, et al. Facing terminal ileitis: going beyond Crohn's disease. Gastroenterology Res 2016; 9: 1-9.

26. Puylaert CAJ, Tielbeek JAW, Schüffler PJ, et al. Comparison of contrast-enhanced and diffusion-weighted MRI in assessment of the terminal ileum in Crohn's disease patients. Abdom Radiol (NY) 2019; 44: 398-405.

27. Buisson A, Pereira B, Goutte M, et al. Magnetic resonance index of activity (MaRIA) and Clermont score are highly and equally effective MRI indices in detecting mucosal healing in Crohn's disease. Dig Liver Dis 2017; 49: 1211-1217. 